\title{
RESEARCH
}

\section{Systematic population-based identification of NTRK and RET fusion-positive thyroid cancers}

\author{
Markus Eszlinger ${ }^{1,2}$, Paul Stewardson ${ }^{3}$, John B McIntyre4, Adrian Box ${ }^{5}$, Moosa Khalil5, Martin Hyrcza ${ }^{5}$, \\ Konstantin Koro5, Dean Ruether ${ }^{6}$, Jiahui Wu ${ }^{3}$ and Ralf Paschke ${ }^{7}$
}

\begin{abstract}
1Departments of Oncology, Pathology and Laboratory Medicine, Biochemistry and Molecular Biology, and Arnie Charbonneau Cancer Institute, Cumming School of Medicine, University of Calgary, Heritage Medical Research Building, Calgary, Alberta, Canada

2Institute of Pathology, University Hospital Halle (Saale), Halle (Saale), Germany

3Department of Medical Science and Arnie Charbonneau Cancer Institute, Cumming School of Medicine, University of Calgary, Calgary, Canada

4Precision Oncology Hub Laboratory, Alberta Health Services, Tom Baker Cancer Centre, Calgary, Alberta, Canada

${ }^{5}$ Department of Pathology and Laboratory Medicine, Cumming School of Medicine, University of Calgary, Calgary, Canada

${ }^{6}$ Section of Medical Oncology, Department of Oncology, Cumming School of Medicine, University of Calgary, Calgary, Canada

'Departments of Medicine, Oncology, Pathology and Laboratory Medicine, Biochemistry and Molecular Biology, and Arnie Charbonneau Cancer Institute, Cumming School of Medicine, University of Calgary, Heritage Medical Research Building, Calgary, Alberta, Canada
\end{abstract}

Correspondence should be addressed to R Paschke: ralf.paschke@ucalgary.ca

\begin{abstract}
Objective: The aim of the study was to identify patients with NTRK fusion-positive or RET fusion/mutation-positive thyroid cancers, who could benefit from neurotrophic tyrosine kinase receptor (NTRK) or receptor tyrosine kinase (RET) inhibitors.

Methods: Patients were identified in the Calgary prospective thyroid cancer database $(N=482)$. Patients were 'pre-screened' with clinically available MassARRAY ${ }^{\circledR}$ BRAF test, Colon Panel, Melanoma Panel, or ThyroSPEC ${ }^{\text {TM. }}$. Mutation-negative tumors were 'screened' for NTRK fusions and RET fusions/mutations with the Oncomine ${ }^{\mathrm{TM}}$ Comprehensive Assay v3 (OCAv3).

Results: A total of 86 patients were included in 1 of 2 separate analyses. Analysis A included 42 patients with radioactive iodine (RAl)-resistant distant metastases. After pre-screening, 20 BRAF and RAS mutation-negative patients underwent OCAv3 screening, resulting in the detection of 4 patients with NTRK fusions and 4 patients with RET fusions (8/20, $40 \%$ of analyzed patients). Analysis B included 44 patients, 42 with American Thyroid Association (ATA) high and intermediate risk of recurrence and 2 with medullary thyroid carcinoma. During pre-screening, 1 patient with an NTRK fusion, 1 patient with a $R E T$ fusion, and 30 patients with BRAF mutations were identified. The remaining 9 patients received OCAv3 screening, resulting in detection of 1 patient with an NTRK fusion and 1 with a RET fusion (4/11, 36\% of analyzed patients).

Conclusions: Our findings indicate a higher rate of NTRK fusions and RET fusions in patients with thyroid cancer with RAI-resistant distant metastases and ATA high or intermediate risk of recurrence. This highlights the importance of early screening to enable intervention with a NTRK or RET inhibitor.
\end{abstract}




\section{Introduction}

The neurotrophic tyrosine kinase receptor (NTRK) inhibitors, larotrectinib and entrectinib, were approved by the United States Food \& Drug Administration (FDA) for the treatment of NTRK fusion-positive solid tumors that are metastatic, where surgery would result in severe morbidity, or where no satisfactory alternative therapy is available. The receptor tyrosine kinase (RET) inhibitors, selpercatinib and pralsetinib, were approved by the FDA for advanced or metastatic RET mutation-positive medullary thyroid cancer (MTC) or RET gene fusion-positive thyroid cancer. The early approval of these drugs was based on phase $1 / 2$ data $(1,2,3,4,5)$. Patients with thyroid cancer were one of the larger patient groups in the larotrectinib basket trial, and the selpercatinib study was conducted entirely in patients with thyroid cancer $(1,3,4,5)$. Five patients with NTRK fusion-positive thyroid cancer were included in the entrectinib phase $1 / 2$ trials and one had a response (6). Both larotrecinib and selpercatinib showed marked and durable overall response rates $(1,3,4,5)$. For the first time for thyroid cancer, complete response was demonstrated in 7\% of the patients with NTRK fusion-positive, larotrectinibtreated, metastatic or locally advanced thyroid cancer, $9 \%$ of the patients with previously treated, RET mutationpositive, selpercatinib-treated MTC, and $5 \%$ of the patients with previously treated RET gene fusion-positive, selpercatinib-treated thyroid cancer $(5,7)$. Only $2 \%$ of selpercatinib- and larotrectinib-treated patients and $4 \%$ of entrectinib-treated patients discontinued the treatment because of treatment-related adverse events $(4,5,6)$. This compares favorably with vandetanib, cabozantinib, or lenvatinib that showed drug discontinuation rates of 21,8 , and 20\%, respectively (8). Further, TRK and RET inhibitors are currently being tested in clinical trials $(4,9)$. The very specific and selective activity profile of these drugs requires stratification of patients for these therapies based on detection of NTRK gene fusions and RET gene fusions/ mutations, respectively.

The prevalence of NTRK fusion and RET fusion has been reported in several prior studies. In The Cancer Genome Atlas (TCGA) study, NTRK fusions and RET fusions were found in 1.2 and $6.8 \%$ of mostly non-metastatic thyroid cancers (10). Across 13 TCGA-analyzed tumor types, thyroid cancer displayed the highest percentage of tumors with druggable kinase fusions at $8.7 \%$ (11). In a Massachusetts General Hospital series of primary thyroid carcinoma, NTRK fusions were found in $3.1 \%$ of cases and 6/11 patients with NTRK fusions had distant metastases (12). In a series of papillary thyroid carcinomas (PTCs),
NTRK fusions were found in $12.6 \%$ of patients and RET gene fusions in $14.3 \%$ (13).

The prevalence of fusions increases for metastatic/ advanced patients' WT of other aberrations. In radioactive iodine (RAI)-resistant metastatic thyroid cancers without $B R A F$, NRAS, HRAS, or KRAS mutations, gene fusions were previously reported in $12 \%$ of 60 samples (4 NTRK fusions, 2 RET fusions, 1 ALK fusion) (14). In patients with BRAF mutation-negative PTC and distant metastases, $67 \%$ had $A L K, N T R K$, or RET fusions (15). In advanced metastatic MTC, the prevalence of $R E T$ mutations was reported as $90 \%$ (16).

There are several recent reviews of the pros and cons for different detection methods $(17,18)$ and for the treatment of kinase fusion-positive cancers (19). However, appropriate and cost-effective screening algorithms to identify and stratify patients with kinase fusionpositive cancers still need to be developed for specific cancer types. A Canadian consensus for adult patients recommends NTRK1-3 gene fusion testing at diagnosis in unresectable or metastatic/advanced patients with all thyroid histologies and at recurrence after surgery \pm RAI treatment if not already performed (20). However, since resensitization for RAI treatment (21) and treatment with the RET inhibitor, selpercatinib (5), are additional options for these patients, integrated molecular stratification strategies are required that allow stratification for all currently available treatment options.

We used a stepwise, recurrence risk-adapted, prioritization approach. This approach had a stepwise application of molecular screening tests, with increasing spectrum of mutation coverage to identify patients with thyroid cancer who could benefit from these new, highly selective, and effective drugs with low adverse event rates. We report the results of this approach in a systematic, integrated, population-based study of patients with thyroid cancer. Our aim was to identify patients with NTRK fusionpositive thyroid cancer and RET fusion/mutation-positive thyroid cancer who can benefit from treatment with NTRK and RET inhibitors.

\section{Materials and methods}

Alberta Health Services is a comprehensive, integrated, single-payer healthcare system with centralized laboratory, pathology, surgery, endocrinology, and oncology services. It has a single electronic medical record system for over 1.5 million inhabitants of the Calgary and Southern Alberta Healthcare regions. The patients for the NTRK fusion and RET fusion/mutation screening were identified in the Calgary

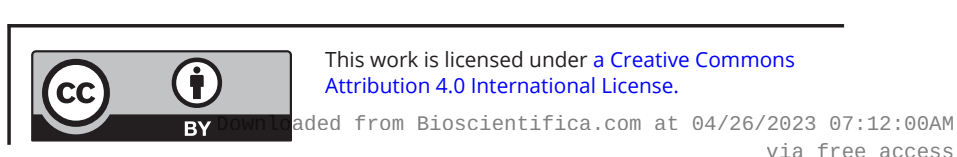


prospective thyroid cancer database, which contains ethics approved research-consented patients with a new diagnosis of thyroid cancer in the Calgary and Southern Alberta Healthcare regions since April 2017. As of December 2020, the Calgary prospective thyroid cancer database contained 482 patients, reflecting a consenting rate of $55.9 \%$ of 862 patients with thyroid cancer since April 2017 with American Thyroid Association (ATA) high $(n=87)$, ATA intermediate $(n=126)$, and ATA low $(n=256)$ recurrence risk and noninvasive follicular thyroid neoplasm with papillary-like nuclear features or MTC patients $(n=13)$ (230 new patients/ year). All new patients with thyroid cancer in the Calgary and Southern Alberta Healthcare regions are prospectively assessed for their ATA recurrence risk, TNM (tumor-nodesmetastases)/MACIS (distant metastasis, patient age, completeness of resection, local invasion, and tumor size) staging, and their indication for RAI treatment, by the thyroid cancer triage group of the University of Calgary, Division of Endocrinology. The study was approved by the University of Calgary's ethical committee. Consent has been obtained from each patient after full explanation of the purpose and nature of all procedures used.

A total of 86 patients were included in 1 of 2 separate analyses we will refer to as Analysis A and Analysis B. Analysis A focused on the primary tumors of 42 patients with RAIresistant distant metastases, identified in June 2020 (Fig. 1). These 42 patients comprise all patients with RAI-resistant distant metastases in the Calgary and Southern Alberta Healthcare regions diagnosed and either treated with Lenvatinib or observed for evidence of progression in June
2020 by the 2 medical oncologists and 2 endocrinologists treating patients with RAI-resistant distant metastases in the Calgary and Southern Alberta Healthcare regions. Sixteen patients with RAI-resistant distant metastases who were diagnosed with thyroid cancer since April 2017 were covered by the thyroid cancer database. Further 3 patients who were not consented until June 2020 and 23 patients who were diagnosed with RAI-resistant distant metastases in the Calgary and Southern Alberta Healthcare regions before April 2017 were identified by capturing all patients with thyroid cancer treated by the 2 medical oncologists and 2 endocrinologists treating patients with RAI-resistant distant metastases in the Calgary and Southern Alberta Healthcare regions.

Sixteen of the 42 patients were on treatment with lenvatinib. One patient in whom lenvatinib was discontinued was subsequently identified as NTRK fusionpositive. To enrich the Oncomine ${ }^{\text {tw }}$ Comprehensive Assay v3 (OCAv3) screening for NTRK fusions and RET fusions/ mutations, the primary tumors were first 'pre-screened" with the successively clinically available MassARRAY ${ }^{\circledR}$ tests, BRAF test, Colon Panel, Melanoma Panel, or ThyroSPEC ${ }^{\text {m }}$ test, with increasing the mutation coverage respectively in addition to BRAF to identify BRAF mutationnegative patients. The mutation spectrum analyzed by these MassARRAY tests in addition to BRAF is given in Supplementary Table 1 (see section on supplementary materials given at the end of this article).

Analysis B targeted the primary tumors of separate 44 patients out of the 482 patients in the Calgary

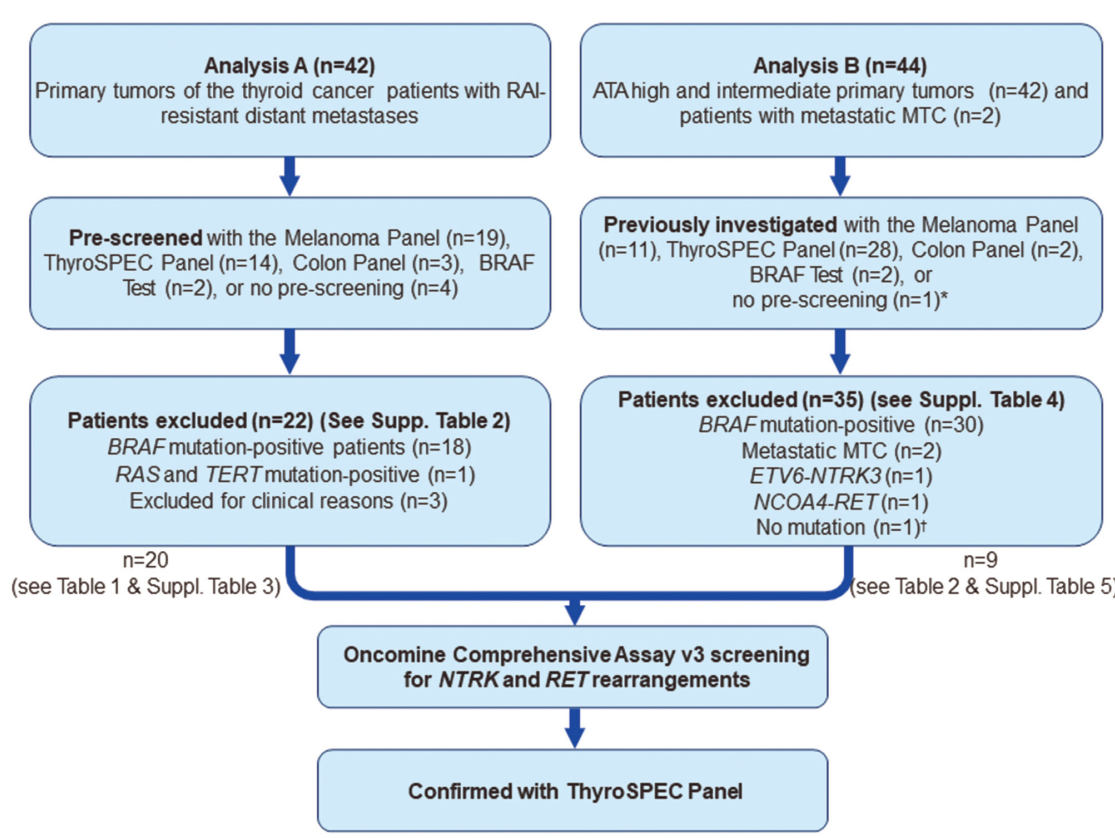

Figure 1

NTRK and RET gene fusion screening.

$$
\begin{array}{lr}
\text { https://etj.bioscientifica.com } & \text { ○ } 2022 \text { The authors } \\
\text { https://doi.org/10.1530/ETJ-21-0061 } & \text { Published by Bioscientifica Ltd. }
\end{array}
$$

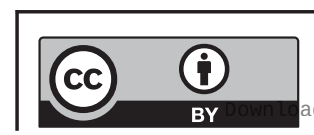

This work is licensed under a Creative Commons Attribution 4.0 International License. ded from Bioscientifica.com at 04/26/2023 07:12:0๑AM 
prospective thyroid cancer database (Fig. 1). These 44 patients included 39/87 ATA high and 3/126 ATA intermediate recurrence risk patients whose primary tumors had previously been investigated with one of the MassARRAY tests. Performance of MassARRAY for the ATA intermediate- and ATA high-risk patients was dependant on the assessment by the six members of the thyroid cancer triage group of the University of Calgary, Division of Endocrinology treating nearly all patients with thyroid cancer in the Calgary and Southern Alberta Healthcare regions. The criteria for the use of MassArray assessment evolved based on the progressive availability of the four MassArray tests described in Supplementary Table 1, case discussions during monthly interdisciplinary thyroid cancer rounds and thyroid cancer treatment pathway evolutions and updates discussed during the meetings of the interdisciplinary provincial endocrine tumor team.

In addition, 2 patients with metastatic MTC were included in these 44 patients.

\section{Oncomine comprehensive assay analysis}

DNA and RNA were extracted from formalin-fixed paraffinembedded (FFPE) tissues using the RecoverAll Total Nucleic Acid Isolation Kit (Thermo Fisher Scientific) according to the manufacturer's protocol. DNA and RNA were quantified using Qubit ${ }^{\mathrm{T}}$ DNA HS assay and Qubit RNA HS assay, respectively. Samples were sequenced using the OCAv3 Chef kit (Thermo Fisher Scientific). Briefly, amplicon libraries were prepared on the Ion Chef instrument using 20 ng DNA input and 22 ng RNA input. RNA was reverse transcribed using SuperScript VILO IV (Thermo Fisher Scientific) according to the manufacturer's protocol. DNA and RNA fusion libraries were combined at an 80:20 ratio (DNA:RNA) and then diluted to a final concentration of $50 \mathrm{pM}$. Sample libraries were then templated and amplified on ion sphere particles and loaded on to 540 chips using the Ion 540 Chef Kit. Each 540 chip multiplexed seven samples and one no template control. Samples were sequenced on the Ion S5 XL sequencer. Data were analyzed using Ion Torrent Suite software v5.14, and variant calling was performed with Ion Reporter v5.16 Oncomine comprehensive v3 workflow 4.1. All variant calls were manually reviewed using Integrated Genomics Viewer.

\section{BRAF, Colon Panel, Melanoma Panel, and ThyroSPEC assay analysis}

DNA and RNA were extracted from FFPE tissues using the AllPrep DNA/RNA FFPE kit (Qiagen), and RNA was reverse transcribed using Qiagen's miScript II kit. Mutation and fusion profiling was performed with Agena Bioscience (San Diego, CA, USA) using iPLEX ${ }^{\circledast}$ Pro (ThyroSPEC, Calgary, Alberta, Canada), iPLEX ${ }^{\circledast}$ HS Colon, or iPLEX ${ }^{\circledast}$ HS Melanoma chemistry on the Agena Bioscience MassARRAY system, with all variant calls reviewed manually using Typer software. All kits were used according to the manufacturer's protocol.

The genotyping process was performed after PCR amplification using a single-base extension technique and mass spectrometry on the MassARRAY instrument. The sensitivity of these assays is $5 \%$, although a conservative $10 \%$ minimum frequency threshold is used for ThyroSPEC.

The genomic alterations each of these tests cover are provided at the following web addresses: Alberta Precision Laboratories | Lab Services (https://www. albertahealthservices.ca/webapps/labservices/indexAPL. asp?id=8557\&tests=\&zoneid=1\&details=true), Agena Melanoma Panel v1.1 (https://www.albertahealthservices. ca/assets/wf/lab/if-lab-hp-cal-agena-melanomapanel-v1-0.pdf), Agena Colon Panel v1.0 (https://www. albertahealthservices.ca/assets/wf/lab/if-lab-hp-calagena-colon-panel-v1.pdf), and ThyroSPEC Molecular Testing of Indeterminate FNA Cytologies (https://www. albertahealthservices.ca/assets/info/hp/cancer/if-hpcancer-guide-molecular-fna-report.pdf).

\section{Results}

Analysis A included 42 thyroid cancer patients with RAI-resistant distant metastases. Pre-screening of these 42 patients led to exclusion of 22 patients from further OCAv3 analysis (Fig. 1). Of these 22 patients, 18 primary tumors were $B R A F$ mutation-positive, 1 had an HRAS mutation and a TERT mutation, and 3 patients were excluded because of clinical reasons (Fig. 1 and Supplementary Table 2).

This pre-screening led to the identification of $20 B R A F$ mutation-negative patients with RAI-resistant distant metastases (Fig. 1 and Supplementary Table 3) who were further analyzed using the OCAv3 panel. We detected 4 RET gene fusions (20\%) and 4 NTRK fusions (20\%) in the $20 B R A F$ mutation-negative patients with RAI-resistant distant metastasis in Analysis A (Table 1). Of the 42 patients with RAI-resistant distant metastases, actionable fusions were ultimately identified in 19\% of patient tumors (Fig. 2).

Twelve of the 87 ATA high recurrence risk patients in the database had distant metastases and were included in the Analysis A of patients with RAI-resistant distant metastases. Comparison of the 42 pre-screened patients with ATA high recurrence risk with the 36 not pre-screened ATA high recurrence risk patients (87 (ATA high-risk

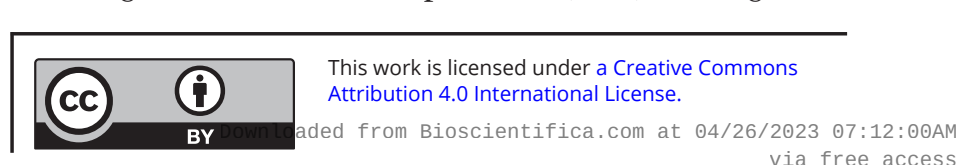


Table 1 Analysis A: Result of first OCAv3a analysis for 20 BRAF mutation-negative patients with RAI-resistant distant metastasis.

\begin{tabular}{|c|c|}
\hline OCAv3 test results & Gene (number of mutations) (histology) \\
\hline \multirow{5}{*}{ RET fusions $(n=4)$} & RET fusion only (3) \\
\hline & CCDC6-RET (dsPTCb) \\
\hline & NCOA4-RET (PTC not specified) \\
\hline & NCOA4-RET (dsPTC) \\
\hline & NCOA4-RET \& TERT C.1-124C>T (1) (PTC solid variant) \\
\hline \multirow[t]{5}{*}{ NTRK fusions $(n=4)$} & NTRK fusion only (3) \\
\hline & SQSTM1-NTRK1 (PTC classic) \\
\hline & EML4-NTRK3 (mixed histology (classic and follicular variant PTC)) \\
\hline & ETV6-NTRK3 (PTC clear cell variant) \\
\hline & ETV6-NTRK3 \& MYCNOS \& TERT (1) (mixed histology (classic and follicular variant PTC)) \\
\hline \multirow[t]{9}{*}{ Other mutations $(n=11)$} & RAS only (2) (FTC minimally invasive, PTC tall cell variant) \\
\hline & RAS \& TERT (2) (PTC oncocytic variant, PTC not specified) \\
\hline & PTEN only (1) (PTC poorly differentiated) \\
\hline & TERT only (1) (PTC oncocytic variant) \\
\hline & PTEN \& TERT (1) (Hurthle cell carcinoma) \\
\hline & MAP2K1 \& TERT (1) (PTC not specified) \\
\hline & RAS \& PIK3CA (1) (FTCc widely invasive) \\
\hline & PIK3CA \& EGFR \& RAS (1) (PTC follicular variant) \\
\hline & RAS \& AKT1 \& TERT (1) (PTC poorly differentiated) \\
\hline No mutation $(n=1)$ & FTC widely invasive \\
\hline
\end{tabular}

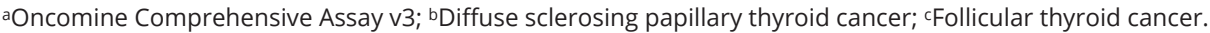

patients in database) - 12 (with distant metastases) - 39 (who were pre-screened) $=36$ (ATA high risk recurrence, not pre-screened)) showed no significant differences for the percentage of excellent response, and the percentages of indeterminate response, structural incomplete response, and biochemical incomplete response, or the mean and median follow-up time.

Analysis B included 44 patients distinct from those included in Analysis A; 42 of these patients were ATA high and intermediate recurrence risk and 2 of these patients had metastatic MTC. Thirty-five of the 44 patients were excluded from OCAv3 analysis due to the detection of $B R A F$ mutations in 30 patients and ThyroSPEC detection of 1 ETV6-NTRK3 fusion in an ATA high-recurrence risk patient with indeterminate response to treatment at 53 months after total thyroidectomy, 1 NCOA4-RET gene fusion in an ATA high-recurrence risk patient with indeterminate response to treatment, and 2 RET point mutations (p.C620R and p.A883F), in the 2 patients with metastatic MTCs (Fig. 1 and Supplementary Table 4).

The primary tumors of the remaining nine patients in Analysis B were selected for further OCAv3 analysis (Supplementary Table 5).

Out of the nine patients who received OCAv3 screening in Analysis B, we detected one NTRK fusion (11\%) and one RET fusion (11\%) (Table 2). Of the 42 ATA high- and intermediate-risk patients and 2 patients with MTC, RET mutations, RET/PTC fusions, or NTRK fusions were ultimately detected in $14 \%$ (Fig. 3 ).
All the OCAv3-detected RET and NTRK fusions detected in tumors that were pre-screened with the successively clinically available MassARRAY ${ }^{\varpi}$ tests, BRAF test, Colon Panel, and Melanoma Panel test, are covered by the ThyroSPEC assay and were subsequently confirmed with the clinically validated ThyroSPEC assay after their detection by OCAv3 analysis. One fusion-positive OCAv3analyzed patient with RAI-resistant distant metastases was found to be ThyroSPEC mutation-negative in the prescreening of a $>15$-year-old FFPE sample. Repeat ThyroSPEC testing with a more recent sample confirmed the ETV6NTRK3 fusion detected by OCAv3.

\section{Discussion}

The two most important results of this systematic approach to identify patients with NTRK fusion and RET fusionpositive thyroid cancers can be summarized as follows:

1. For $20 B R A F$ mutation-negative patients, among 42 patients with RAI-resistant metastatic thyroid cancer, the OCAv3 analysis detected 4 NTRK fusions and 4 RET fusions. This $40 \%$ prevalence of NTRK fusion or $R E T$ fusion positivity for BRAF mutation-negative RAI-resistant metastatic thyroid cancers significantly contrasts with the previously reported $12 \%$ prevalence (4 NTRK fusions, 2 RET fusions, and $1 A L K$ fusion) for 60 BRAF mutation-negative patients (Pearson's Chisquared test, $P=0.013$ ) (14).

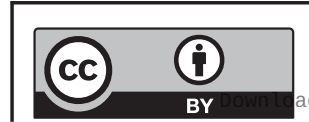

This work is licensed under a Creative Commons Attribution 4.0 International License. 
A

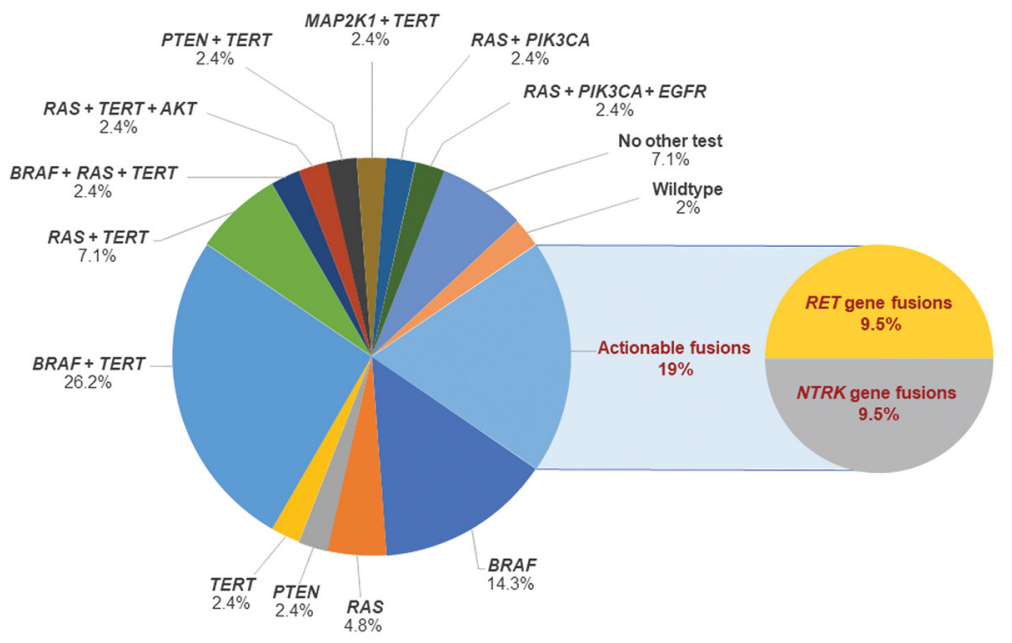

B

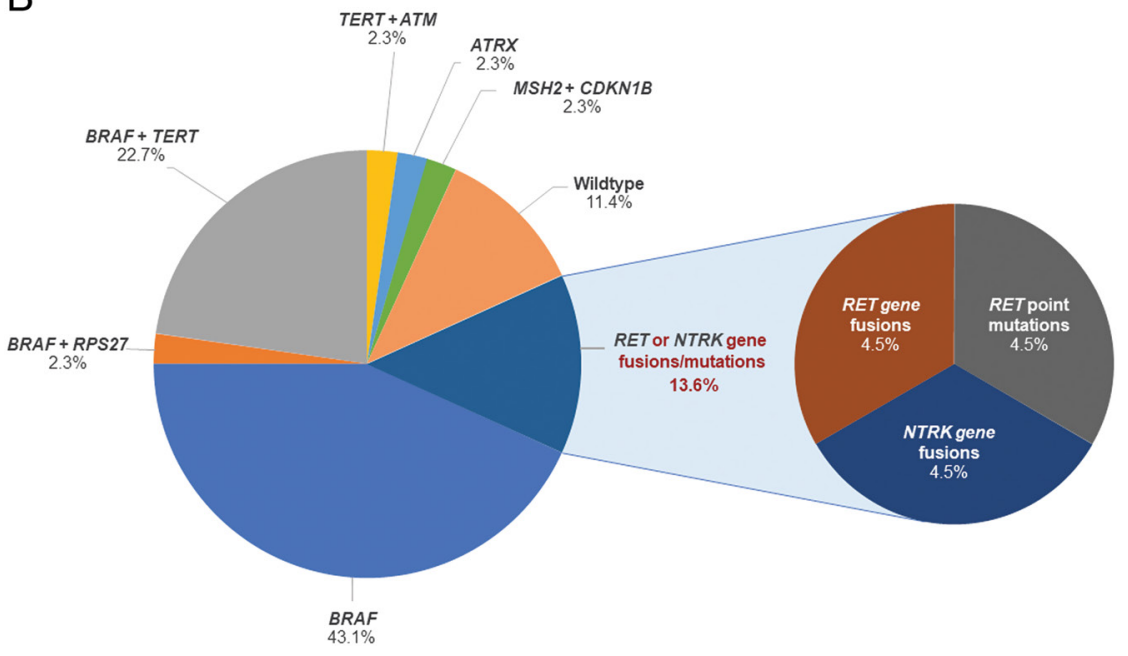

\section{Figure 2}

(A) Mutations and fusions detected in 42 patients with radioiodine-resistant distant metastasis. (B) Mutations and fusions detected in 42 American Thyroid Association (ATA) high and intermediate risk patients and 2 patients with medullary thyroid cancer (MTC).

Table 2 Analysis B: Result of OCAv3a analysis for nine patients with ATA high or intermediate recurrence risk and no BRAF mutation, RET mutation, NTRK fusion, or RET fusion in the pre-screening.

\begin{tabular}{|c|c|}
\hline OCAv3 test results & Mutated gene (number of mutations) \\
\hline$R E T$ fusion $(n=1)$ & $\begin{array}{l}\text { RET fusion only (1) } \\
\quad \text { CCDC6-RET }\end{array}$ \\
\hline NTRK fusion $(n=1)$ & $\begin{array}{l}\text { NTRK fusion only (1) } \\
\text { TPM3-NTRK1 (selected based on dsPTC b histology) }\end{array}$ \\
\hline Other mutations $(n=3)$ & $\begin{array}{l}\text { ATRX only (1) (PTC poorly differentiated) } \\
\text { MSH2 \& CDKN1B (1) (PTC poorly differentiated) } \\
\text { ATM \& TERT (1) (PTC poorly differentiated) }\end{array}$ \\
\hline No mutation $(n=4)$ & $\begin{array}{l}\text { Hurthle cell carcinoma (1) } \\
\text { PTC tall cell (1) } \\
\text { PTC classic (1) } \\
\text { PTC follicular variant (1) }\end{array}$ \\
\hline
\end{tabular}

aOncomine Comprehensive Assay v3; bDiffuse sclerosing papillary thyroid cancer.

https://etj.bioscientifica.com

https://doi.org/10.1530/ETJ-21-0061 (c) 2022 The authors Published by Bioscientifica Ltd.

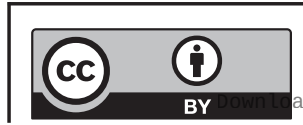

This work is licensed under a Creative Commons Attribution 4.0 International License.

ded from Bioscientifica.com at 04/26/2023 07:12: ๑०AM 


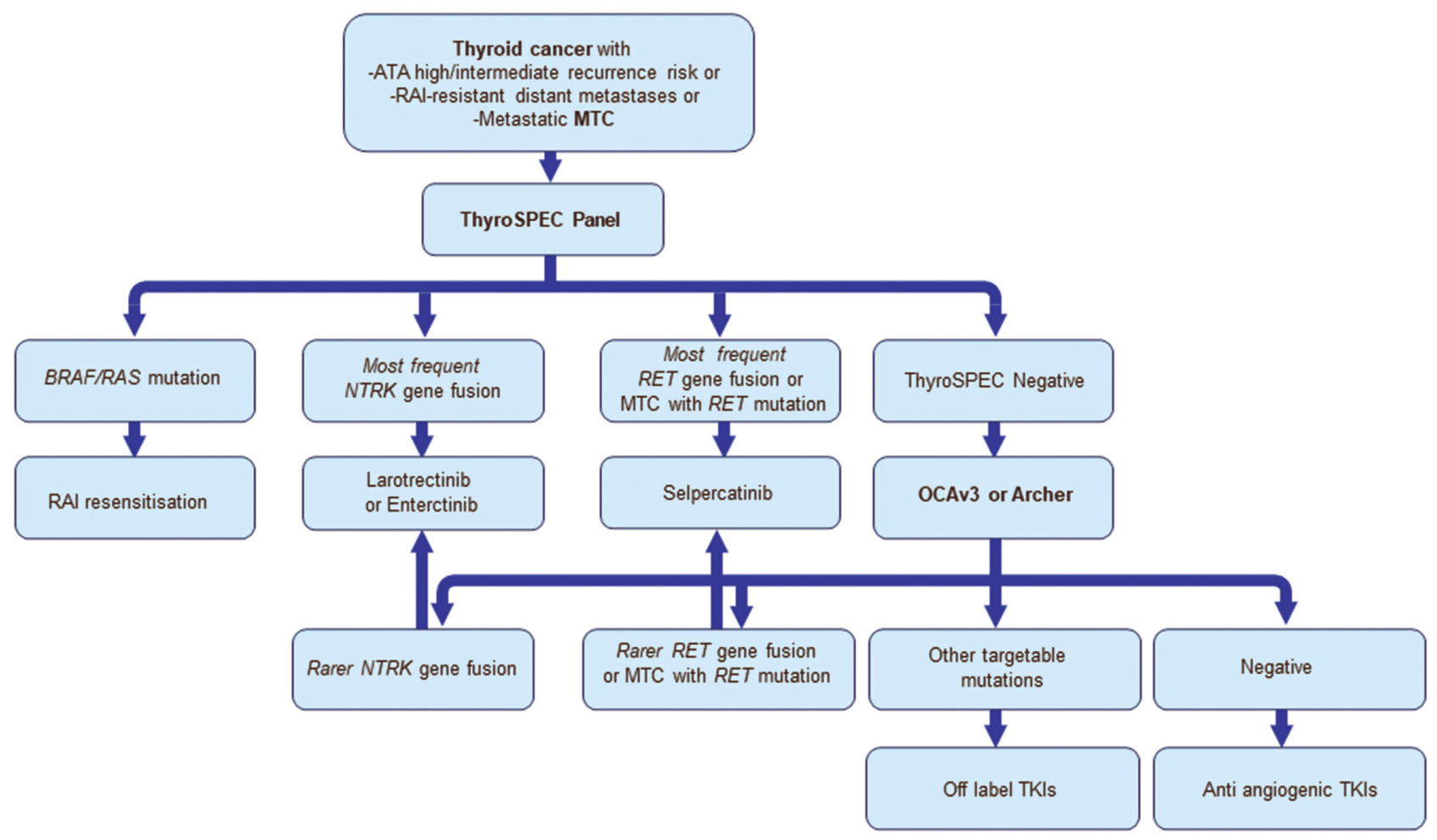

Figure 3

Proposed testing and treatment algorithm for Alberta Health Services.

2. In 11 BRAF mutation-negative patients among 42 patients with ATA high and intermediate recurrence risk follicular thyroid cancers, the OCAv3 and ThyroSPEC analyses detected 2 NTRK fusions and 2 RET fusions (36\%) (2 identified during pre-screening with ThyroSPEC and 2 identified during subsequent OCAv3 analysis).

The results of our systematic screening strategy suggest a much higher rate of NTRK fusions and RET fusions in our pre-selected patient groups than previously reported in the TCGA study with mostly non-metastatic thyroid cancers or for RAI-resistant metastatic thyroid cancers without $B R A F$, NRAS, HRAS, or KRAS mutations $(10,14)$. Our results align closer to the more recent series of patients from Lan and colleagues, where $67 \%$ of $B R A F$ mutation-negative patients with PTC with distant metastases were found to have gene fusions (15).

Most important, these results suggest that all $B R A F$ mutation-negative patients with RAI-resistant metastatic thyroid cancer should be screened for NTRK fusions and RET fusions to stratify these patients early for treatment with the NTRK and RET inhibitors larotrectinib, entrectinib, and selpercatinib, pralsetinib. If NTRK fusions and RET fusions are identified, these patients could benefit from higher and more sustained response rates and a much lower burden of adverse events than current standard of care.

Furthermore, this study demonstrates for the first time that all $B R A F$ mutation-negative patients with progressive structural incomplete response to total thyroidectomy and RAI treatment and ATA high- or intermediate-recurrence risk patients with progressive structural incomplete response should be screened for NTRK fusions and RET fusions to enable early intervention. These patients require early stratification for cutting-edge treatment options, since $56-72$ and $21-34 \%$ of patients with high or intermediate risk of recurrence patients, respectively, have persistent/recurrent structural disease a median of 4-10 years after total thyroidectomy and RAI (22, 23, 24, 25). Revision surgery and/or a second RAI treatment is largely unsuccessful and will only lead to remission in up to $51 \%$ of these patients $(26,27,28)$.

Since all the OCAv3-detected RET fusions and NTRK fusions were subsequently confirmed with the ThyroSPEC Panel, future primary molecular stratifications of progressive RAI-resistant metastatic and ATA high recurrence risk thyroid cancers with structural incomplete response in the Alberta Healthcare regions will be performed with the ThyroSPEC Panel. Aberration-negative samples should still be further investigated with broader tests like OCAv3 or Archer 
FusionPlex Thyroid and Lung to account for the rarer fusions with DIAPH1, EML4, IRF2BP2, NFASC, and PPL, which were NTRK partners reported in the larotrectinib phase 1 and 2 trials and ETV6, EML4, RBPMS, SQSTM1, TPM3, IRF2BP2, SQSTM1, and TPR detected in a recent NTRK rearrangement detection study that are not included in the ThyroSPEC Panel and CCDC186, ERC1, KTN1, and RUFY3, which were RET partners reported in the selpercatinib trial not included in the ThyroSPEC Panel $(4,5,29,30)$. This approach will stratify $B R A F$ mutation-positive patients for re-expression of the iodine symporter with dabrafenib and trametenib to re-enable RAI treatment. Moreover, this approach will simultaneously identify most patients eligible for treatment with larotrectinib, entrectinib and selpercatinib, pralsetinib. Thus, very few patients will require analysis with more comprehensive mutation and gene fusion panels to provide access to currently available drugs (Fig. 3).

A recent international expert consensus recommends that all patients with advanced (unresectable or metastatic) solid tumors without actionable driver gene mutations/ fusions/amplifications should be tested for NTRK fusions, especially ETV6-NTRK3 fusions in tumors with a high incidence of NTRK fusions (19). Also, patients with locally advanced tumors with a high incidence of NTRK fusions should be tested when considering neoadjuvant therapy before resection (19). This testing should be performed before or during the standard treatment of advanced solid tumors (19). Our results support these recommendations and add further detail to these recommendations for thyroid cancer. The Canadian consensus for adult patients recommends NTRK1-3 fusion testing at diagnosis in unresectable or metastatic/advanced patients with all thyroid histologies and at recurrence after surgery \pm RAI if not already performed (20). While these recommendations focused on NTRK inhibitors, our integrated molecular strategy allows stratification for all currently available treatment options and includes resensitization for radioiodine treatment or treatment with the RET inhibitor selpercatinib $(5,21)$.

Patients with metastatic dsPTC ( 2 of 20 OCAv3-screened patients in Analysis A and 1 of 9 OCAv3-screened patients within Analysis B) show a very high yield for RET fusions $(2 / 3,66 \%)$. This is in line with a previous report by Joung and colleagues, which demonstrated for 37 cases of dsPTC, 17 were positive for RET/PTC1 (46\%) and 6 for RET/PTC3 (16\%) (31).

The OCAv3 analysis also detected further mutations summarized in Table 3 with potential relevance for off-label

Table 3 Mutations detected with OCAv3a with potential relevance for off-label drug use based on current clinical trials.

$\begin{array}{ll}\begin{array}{l}\text { Mutations (VAFb) } \\ \begin{array}{c}\text { PTEN R130L } \\ (27 \%)\end{array}\end{array} & \text { ClinVar interpretation (25) } \\ \begin{array}{ll}\text { PIK3CA H1047R } \\ (23 \%)\end{array} & \text { Pathogenic } \\ \text { EGFR P848L (52\%) } & \begin{array}{l}\text { Likely benign/unknown } \\ \text { significance }\end{array} \\ \text { MSH2 Q510* } & \begin{array}{c}\text { Pathogenic } \\ (73 \%)\end{array}\end{array}$

CDKN1B S7* (8\%) Uncertain significance

ATM S151* (85\%) Uncertain significance

ATRX E680* (42\%) No entry

\begin{tabular}{l}
\hline OncoKB \\
interpretation (26) \\
\hline Oncogenic
\end{tabular}

Oncogenic

Likely neutral

Unknown oncogenic effect

\section{OncoKB therapeutic effect (26) \\ Compelling biological evidence \\ supports the biomarker as being} predictive of response to a drug but neither biomarker nor drug is standard of care

FDAc-recognized biomarker predictive of response to an FDA-approved drug in this indication (breast cancer: fulvestrant + alpelisib) $-$

No FDA-approved or NCCNd compendium listed treatments specifically for patients with thyroid cancer with MSH2-truncating mutations.

Unknown oncogenic No FDA-approved or NCCNeffect compendium listed treatments specifically for patients with CDKN1B S7 mutant thyroid cancer.

Unknown oncogenic No FDA-approved or NCCN effect compendium listed treatments specifically for patients with ATM S151 mutant thyroid cancer.

Unknown oncogenic No FDA-approved or NCCN effect compendium listed treatments specifically for patients with ATRX E680 mutant thyroid cancer.

\begin{tabular}{l} 
Clinical trials \\
\hline NCT02465060 \\
NCT02465060 \\
- \\
- \\
..
\end{tabular}

28 ongoing studies for patients with ATM-mutated solid tumors NCT03718091$$
\text { 政 }
$$
.

aOncomine Comprehensive Assay v3; bVariant allele frequency; cFood \& Drug Administration; dNational Comprehensive Cancer Network.

https://etj.bioscientifica.com

https://doi.org/10.1530/ETJ-21-0061 (c) 2022 The authors Published by Bioscientifica Ltd.

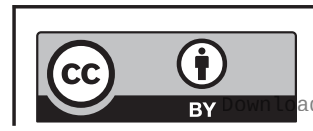

This work is licensed under a Creative Commons Attribution 4.0 International License. ded from Bioscientifica.com at 04/26/2023 07:12: ๑๑AM via free access 
drug use based on current clinical trials (clinicaltrials.gov) (32). The future treatment and follow-up of the respective patients and availability of new drugs will determine if patients with negative results for pre-screening tests like ThyroSPEC should undergo more extended mutation screening as currently done for lung cancers in the Lung Cancer Master Protocol (Lung-MAP) $(33,34)$ or multiple myeloma in the Bellini study (35) and other programs.

Possible limitations of this study include the lack of RNAseq as a comprehensivegold standard for rearrangement detection and the past report of concomitant $R A S$ and $B R A F$ or RET/PTC and BRAF mutations in 11 of 88 mostly advanced stage PTCs and in 14 of 72 conventional PTCs (36, 37). Similarly, Yoshihara and colleagues found targetable kinase mutations in $4.9 \%$ of patients with thyroid cancer and RET fusions, $1 \%$ of patients with thyroid cancer with NTRK1 fusions, and $1.8 \%$ of patients with thyroid cancer with NTRK3 fusions (11). Therefore, further data are required to decide if $B R A F$ - and $R A S$-positive patients should be excluded in future screening strategies for RET/PTC or NTRK fusions. However, pre-screening with ThyroSPEC covering the most frequent BRAF, RAS, and $R E T$ point mutations, as well as the most frequent $R E T / \mathrm{PTC}$ and NTRK fusions, may effectively address this question.

In summary, our results demonstrate a high frequency of NTRK fusions and RET fusions/mutations in patients with thyroid cancer with RAI-resistant distant metastases, ATA high, or ATA intermediate risk of recurrence, whose tumors are negative for other mutations/fusions. Our data emphasize the need for genomic testing in these patient populations to identify those who may benefit from effective and well-tolerated therapies, larotrectinib, entrectinib, and selpercatinib.

\section{Supplementary materials}

This is linked to the online version of the paper at https://doi.org/10.1530/ ETJ-21-0061.

\section{Declaration of interest}

A $B$ reports: Advisory board honoraria from AstraZeneca and Bayer. $\mathrm{M} \mathrm{H}$ reports: Advisory board and teaching honoraria from Bayer. D R reports: Advisory board honoraria from Eisai, Ipsen, Novartis, Pfizer. R P reports: Grants from Bayer, and advisory board honoraria from Bayer and Eisai. The other authors have nothing to disclose.

\section{Funding}

The work was funded by Bayer Inc.

\section{Author contribution statement}

M E: study outline and organization, data collection, verification of underlying data and discussion of data, and writing of the manuscript. P S: data collection, verification of underlying data and discussion of data, and writing of the manuscript. J B M: performed the OCAv3 analysis. A B: responsible for melanoma and Colon Panel and ThyroSPEC analysis. M K: performed histology review. $\mathrm{M} \mathrm{H}$ : performed histology review. K K: performed histology review. D R: contributed patients with metastatic thyroid cancer. J W: data collection, verification of underlying data, and statistical analysis. R P: study outline and organization, data collection, verification of underlying data and discussion of data, and writing of the manuscript.

\section{Acknowledgements}

Editorial support was provided by MEDUCOM Health Inc. and sponsored by Bayer Inc. The authors acknowledge Cristi Williamson (MEDUCOM Health Inc., Guelph, ON, Canada) for medical writing services in editing the first draft prepared by the authors.

\section{References}

1 Laetsch TW, DuBois SG, Mascarenhas L, Turpin B, Federman N, Albert CM, Nagasubramanian R, Davis JL, Rudzinski E, Feraco AM, et al. Larotrectinib for paediatric solid tumours harbouring NTRK gene fusions: phase 1 results from a multicentre, open-label, phase 1/2 study. Lancet: Oncology 201819 705-714. (https://doi.org/10.1016/ S1470-2045(18)30119-0)

2 Subbiah V, Gainor JF, Rahal R, Brubaker JD, Kim JL, Maynard M, Hu W, Cao Q, Sheets MP, Wilson D, et al. Precision targeted therapy with BLU667 for RET-driven cancers. Cancer Discovery 20188 836-849. (https:// doi.org/10.1158/2159-8290.CD-18-0338)

3 Hong DS, Bauer TM, Lee JJ, Dowlati A, Brose MS, Farago AF, Taylor M, Shaw AT, Montez S, Meric-Bernstam F, et al. Larotrectinib in adult patients with solid tumours: a multi-centre, open-label, phase I doseescalation study. Annals of Oncology 201930 325-331. (https://doi. org/10.1093/annonc/mdy539)

4 Hong DS, DuBois SG, Kummar S, Farago AF, Albert CM, Rohrberg KS, van Tilburg CM, Nagasubramanian R, Berlin JD, Federman N, et al. Larotrectinib in patients with TRK fusion-positive solid tumours: a pooled analysis of three phase 1/2 clinical trials. Lancet: Oncology 2020 21 531-540. (https://doi.org/10.1016/S1470-2045(19)30856-3)

5 Wirth LJ, Sherman E, Robinson B, Solomon B, Kang H, Lorch J, Worden F, Brose M, Patel J, Leboulleux S, et al. Efficacy of selpercatinib in RET-altered thyroid cancers. New England Journal of Medicine 2020 383 825-835. (https://doi.org/10.1056/NEJMoa2005651)

6 Doebele RC, Drilon A, Paz-Ares L, Siena S, Shaw AT, Farago AF, Blakely CM, Seto T, Cho BC, Tosi D, et al. Entrectinib in patients with advanced or metastatic NTRK fusion-positive solid tumours: integrated analysis of three phase 1-2 trials. Lancet Oncology 202021 271-282. (https://doi.org/10.1016/s1470-2045(19)30691-6)

7 Cabanillas ME, Drilon A, Farago AF, Brose MS, McDermott R, Sohal D, Oh D, Almubarak M, Bauman J, Chu E, et al. 1916P Larotrectinib treatment of advanced TRK fusion thyroid cancer. Annals of Oncology 202031 S1026-S1033. (https://doi.org/10.1016/j. annonc.2020.08.1404)

8 Subbiah V, Yang D, Velcheti V, Drilon A \& Meric-Bernstam F. Stateof-the-art strategies for targeting RET-dependent cancers. Journal of Clinical Oncology 202038 1209-1221. (https://doi.org/10.1200/ JCO.19.02551)

9 Li AY, McCusker MG, Russo A, Scilla KA, Gittens A, Arensmeyer K, Mehra R, Adamo V \& Rolfo C. RET fusions in solid tumors. Cancer Treatment Reviews 201981 101911. (https://doi.org/10.1016/j. ctrv.2019.101911)

10 Cancer Genome Atlas Research Network. Integrated genomic characterization of papillary thyroid carcinoma. Cell 2014159 676-690. (https://doi.org/10.1016/j.cell.2014.09.050)

11 Yoshihara K, Wang Q, Torres-Garcia W, Zheng S, Vegesna R, Kim H $\&$ Verhaak RG. The landscape and therapeutic relevance of cancer-

This work is licensed under a Creative Commons Attribution 4.0 International License. 
associated transcript fusions. Oncogene 201534 4845-4854. (https:// doi.org/10.1038/onc.2014.406)

12 Chu YH, Dias-Santagata D, Farahani AA, Boyraz B, Faquin WC, Nosé V $\&$ Sadow PM. Clinicopathologic and molecular characterization of NTRK-rearranged thyroid carcinoma (NRTC). Modern Pathology 2020 33 2186-2197. (https://doi.org/10.1038/s41379-020-0574-4)

13 Musholt TJ, Musholt PB, Khaladj N, Schulz D, Scheumann GF \& Klempnauer J. Prognostic significance of RET and NTRK1 rearrangements in sporadic papillary thyroid carcinoma. Surgery 2000 128 984-993. (https://doi.org/10.1067/msy.2000.110845)

14 van der Tuin K, Ventayol Garcia M, Corver WE, Khalifa MN, Ruano Neto D, Corssmit EPM, Hes FJ, Links TP, Smit JWA, Plantinga TS, et al. Targetable gene fusions identified in radioactive iodine refractory advanced thyroid carcinoma. European Journal of Endocrinology 2019 180 235-241. (https://doi.org/10.1530/EJE-18-0653)

15 Lan X, Bao H, Ge X, Cao J, Fan X, Zhang Q, Liu K, Zhang X, Tan Z, Zheng $\mathrm{C}$, et al. Genomic landscape of metastatic papillary thyroid carcinoma and novel biomarkers for predicting distant metastasis. Cancer Science 2020111 2163-2173. (https://doi.org/10.1111/cas.14389)

16 Romei C, Ciampi R, Casella F, Tacito A, Torregrossa L, Ugolini C, Basolo F, Materazzi G, Vitti P \& Elisei R. RET mutation heterogeneity in primary advanced medullary thyroid cancers and their metastases. Oncotarget 20189 9875-9884. (https://doi.org/10.18632/ oncotarget.23986)

17 Cocco E, Scaltriti M \& Drilon A. NTRK fusion-positive cancers and TRK inhibitor therapy. Nature Reviews: Clinical Oncology 201815 731-747. (https://doi.org/10.1038/s41571-018-0113-0)

18 Solomon JP, Benayed R, Hechtman JF \& Ladanyi M. Identifying patients with NTRK fusion cancer. Annals of Oncology 201930

19 Yoshino T, Pentheroudakis G, Mishima S, Overman MJ, Yeh KH, Baba E, Naito Y, Calvo F, Saxena A, Chen LT, et al. JSCO-ESMO-ASCOJSMO-TOS: international expert consensus recommendations for tumour-agnostic treatments in patients with solid tumours with microsatellite instability or NTRK fusions. Annals of Oncology 202031 861-872. (https://doi.org/10.1016/j.annonc.2020.03.299)

20 Bebb DG, Banerji S, Blais N, Desmeules P, Gill S, Grin A, Feilotter H, Hansen AR, Hyrcza M, Krzyzanowska M, et al. Canadian consensus for biomarker testing and treatment of TRK fusion cancer in adults. Current Oncology 202128 523-548. (https://doi.org/10.3390/ curroncol28010053)

21 Jaber T, Waguespack SG, Cabanillas ME, Elbanan M, Vu T, Dadu R, Sherman SI, Amit M, Santos EB, Zafereo M, et al. Targeted therapy in advanced thyroid cancer to resensitize tumors to radioactive iodine. Journal of Clinical Endocrinology and Metabolism 2018103 3698-3705. (https://doi.org/10.1210/jc.2018-00612)

22 Tuttle RM, Tala H, Shah J, Leboeuf R, Ghossein R, Gonen M, Brokhin M, Omry G, Fagin JA \& Shaha A. Estimating risk of recurrence in differentiated thyroid cancer after total thyroidectomy and radioactive iodine remnant ablation: using response to therapy variables to modify the initial risk estimates predicted by the new American Thyroid Association staging system. Thyroid 201020 1341-1349. (https://doi.org/10.1089/thy.2010.0178)

23 Vaisman F, Momesso D, Bulzico DA, Pessoa CH, Dias F, Corbo R, Vaisman M \& Tuttle RM. Spontaneous remission in thyroid cancer patients after biochemical incomplete response to initial therapy. Clinical Endocrinology 201277 132-138. (https://doi.org/10.1111/j.13652265.2012.04342.x)

24 Pitoia F, Bueno F, Urciuoli C, Abelleira E, Cross G \& Tuttle RM. Outcomes of patients with differentiated thyroid cancer risk-stratified according to the American Thyroid Association and Latin American viii16-viii22. (https://doi.org/10.1093/annonc/mdz384)

Thyroid Society risk of recurrence classification systems. Thyroid 2013 23 1401-1407. (https://doi.org/10.1089/thy.2013.0011)

25 Haugen BR, Alexander EK, Bible KC, Doherty GM, Mandel SJ, Nikiforov YE, Pacini F, Randolph GW, Sawka AM, Schlumberger M, et al. 2015 American Thyroid Association management guidelines for adult patients with thyroid nodules and differentiated thyroid cancer: the American Thyroid Association guidelines task force on thyroid nodules and differentiated thyroid cancer. Thyroid 201626 1-133. (https://doi.org/10.1089/thy.2015.0020)

26 Yim JH, Kim WB, Kim EY, Kim WG, Kim TY, Ryu JS, Gong G, Hong SJ $\&$ Shong YK. The outcomes of first reoperation for locoregionally recurrent/persistent papillary thyroid carcinoma in patients who initially underwent total thyroidectomy and remnant ablation. Journal of Clinical Endocrinology and Metabolism 201196 2049-2056. (https://doi.org/10.1210/jc.2010-2298)

27 Sabra MM, Grewal RK, Tala H, Larson SM \& Tuttle RM. Clinical outcomes following empiric radioiodine therapy in patients with structurally identifiable metastatic follicular cell-derived thyroid carcinoma with negative diagnostic but positive post-therapy 131I whole-body scans. Thyroid 201222 877-883. (https://doi.org/10.1089/thy.2011.0429)

28 Hirsch D, Gorshtein A, Robenshtok E, Masri-Iraqi H, Akirov A, Duskin Bitan H, Shimon I \& Benbassat C. Second radioiodine treatment: limited benefit for differentiated thyroid cancer with locoregional persistent disease. Journal of Clinical Endocrinology and Metabolism 2018 103 469-476. (https://doi.org/10.1210/jc.2017-01790)

29 Rudzinski E, Hong D, Rudolph M, Lockwood C, Reeves J, Moh A, Nogai H, Laetsch T \& Drilon A. Neurotrophic tyrosine receptor kinase (NTRK) gene fusion testing in clinical trials of larotrectinib. Journal of Molecular Diagnostics 20202275.

30 Pekova B, Sykorova V, Mastnikova K, Vaclavikova E, Moravcova J, Vlcek P, Lastuvka P, Taudy M, Katra R, Bavor P, et al. NTRK fusion genes in thyroid carcinomas: clinicopathological characteristics and their impacts on prognosis. Cancers 202113 1932. (https://doi.org/10.3390/ cancers13081932)

31 Joung JY, Kim TH, Jeong DJ, Park SM, Cho YY, Jang HW, Jung YY, Oh YL, Yim HS, Kim YL, et al. Diffuse sclerosing variant of papillary thyroid carcinoma: major genetic alterations and prognostic implications. Histopathology 201669 45-53. (https://doi.org/10.1111/his.12902)

32 Chakravarty D, Gao J, Phillips SM, Kundra R, Zhang H, Wang J, Rudolph JE, Yaeger R, Soumerai T, Nissan MH, et al. OncoKB: a precision oncology knowledge base. JCO Precision Oncology 20172017 1-16. (https://doi.org/10.1200/PO.17.00011)

33 Redman MW, Papadimitrakopoulou VA, Minichiello K, Hirsch FR, Mack PC, Schwartz LH, Vokes E, Ramalingam S, Leighl N, Bradley J, et al. Biomarker-driven therapies for previously treated squamous nonsmall-cell lung cancer (Lung-MAP SWOG S1400): a biomarker-driven master protocol. Lancet: Oncology 202021 1589-1601. (https://doi. org/10.1016/S1470-2045(20)30475-7)

34 Schuler M. The quest for efficient trial designs in precision oncology. Lancet: Oncology 202021 1539-1541. (https://doi.org/10.1016/S14702045(20)30553-2)

35 Ghobrial I. BELLINI: a renaissance for an era of precision therapy in multiple myeloma. Lancet Oncology 202021 1547-1549.

36 Guerra A, Zeppa P, Bifulco M \& Vitale M. Concomitant BRAF(V600E) mutation and RET/PTC rearrangement is a frequent occurrence in papillary thyroid carcinoma. Thyroid 201424 254-259. (https://doi. org/10.1089/thy.2013.0235)

37 Zou M, Baitei EY, Alzahrani AS, BinHumaid FS, Alkhafaji D, Al-Rijjal RA, Meyer BF \& Shi Y. Concomitant RAS, RET/PTC, or BRAF mutations in advanced stage of papillary thyroid carcinoma. Thyroid 201424 1256-1266. (https://doi.org/10.1089/thy.2013.0610)

Received in final form 13 November 2021

Accepted 10 December 2021

Accepted Manuscript published online 10 December 2021

This work is licensed under a Creative Commons

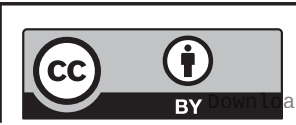
Attribution 4.0 International License. ded from Bioscientifica.com at 04/26/2023 07:12:00AM 